\title{
Effects of Exercise Training After Open Heart Surgery on Quality of Life and Exercise Tolerance in Patients With Mitral Regurgitation or Aortic Regurgitation
}

\author{
Kenji Ueshima, ${ }^{1}$ MD, Junya Kamata, ${ }^{1}$ MD, Noboru Kobayashi, ${ }^{1}$ MD, \\ Masahiko SAITO, ${ }^{1} \mathrm{MD}$, Shigeru SATO, ${ }^{1} \mathrm{PT}$, Kohei KaWAZOE, ${ }^{1} \mathrm{MD}$, \\ and Katsuhiko HIRAMORI, ${ }^{1} \mathrm{MD}$
}

\begin{abstract}
SUMMARY
We conducted a questionnaire survey regarding quality of life (QOL) to evaluate the effects of exercise training on the QOL in patients with valvular heart disease after surgery. This study included 64 consecutive patients who underwent heart surgery. They were divided into two groups: exercise training could $(\mathrm{EX}(+)$ group, $n=31)$ and could not be performed (EX(-) group, $n=33$ ) until 6 months after surgery. To evaluate the QOL and exercise tolerance, we employed "a questionnaire regarding disease and quality of life" developed for Japanese people and cardiopulmonary exercise testing. In our questionnaire survey, the improvement rating ( $\Delta$ subjective/social index), which was calculated from the difference between the pre- and postoperative values, was greater in the $\mathrm{EX}(+)$ group than that in the EX(-) group $(4.9 \pm 3.1$ versus $1.1 \pm 4.0, P<0.05)$. In the changes in exercise tolerance, $\Delta$ anaerobic threshold was greater in the $\mathrm{EX}(+)$ group $(0.79 \pm 0.17$ versus $-0.02 \pm 0.28, P<0.01)$. Moreover, there was a positive correlation between $\Delta$ subjective/social index and $\Delta$ peak $\mathrm{VO}_{2}(\mathrm{r}=0.62, P<0.05)$. We concluded that exercise training for patients after cardiac surgery improves the QOL and exercise tolerance. It is suggested that changes in subjective and social parameters contribute to an exercise training-related improvement in the QOL. (Jpn Heart J 2004; 45: 789-797)
\end{abstract}

Key words: Open heart surgery, Valvular heart disease, Exercise training, Exercise tolerance, Quality of life (QOL)

IT has been reported that exercise training in patients with chronic heart failure improves exercise tolerance, prognosis, and the quality of life (QOL) ${ }^{1-3)}$ However, none of the previous studies have reported the effects of continuous exercise training after open heart surgery in patients with chronic heart failure related to valvular heart disease in Japan. In particular, the role of exercise training in amelioration of the QOL has not been demonstrated.

From the ${ }^{1}$ Iwate Medical University, Memorial Heart Center, Iwate, Japan.

Address for correspondence: Kenji Ueshima, MD, Iwate Medical University, Memorial Heart Center, 1-2-1, Chuoudori, Morioka-shi, Iwate 020-8505, Japan.

Received for publication January 16, 2004

Revised and accepted March 5, 2004. 
In this study, we conducted a questionnaire survey regarding the QOL and cardiopulmonary exercise testing $(\mathrm{CPX})^{4)}$ to evaluate the effects of exercise training in patients with valvular heart disease after open heart surgery on the QOL and exercise tolerance.

\section{Methods}

Subjects: This study included 64 consecutive patients with valvular heart disease who underwent elective open heart surgery (40 males, 24 females, mean age: 58 \pm 5 years, 28 patients with aortic regurgitation, 36 patients with mitral valve regurgitation). The subjects were divided into 2 groups because the study was designed as a nonrandomized case controlled study: patients in whom supervised exercise training could be continuously performed 2-3 times a week after discharge until 6 months were assigned to the exercise training group $(n=31)$, while those who could not come to our rehabilitation office mainly because their place of residence was too far from our institution and/or could not be trained after discharge were assigned to the nonexercise group $(n=33)$. There were no significant differences in clinical features such as age, gender, underlying heart disease, or surgical procedure between the two groups. Furthermore, there were no significant differences in various parameters in the preoperative questionnaire survey regarding the QOL and various parameters on the $\mathrm{CPX}$ and the resting respiratory function test between the two groups (Table).

Supervised exercise training was performed in all patients until discharge (mean training period: 14 days). In the exercise training group, physical training, which was prescribed on the basis of the anaerobic threshold, was performed 2-3 times per week for 6 months. This training was performed in conjunction with electrocardiography and blood pressure monitoring. A questionnaire survey regarding the QOL, the CPX, and the resting respiratory function test was performed before surgery and 1,3, and 6 months after surgery in the exercise group and nonexercise group. Serial changes in these parameters were compared between the two groups.

To evaluate the QOL, we employed "a questionnaire regarding disease and quality of life", ${ }^{5)}$ which consisted of 39 questions prepared by a study group on methods for evaluating the quality of life scales for Japanese patients with cardiovascular diseases, (Ministry of Health and Welfare (Director: Jun Fujii)). We collected and analyzed the data on nonspecific mental/physical symptoms (8 items, 16 points), subjective/social indices (QOL score in a narrow sense; 18 items, 36 points), work (3 items, 6 points), disease-specific symptoms (heart failure; 10 items, 20 points), and total scores (39 items, 78 points). 
Table. Comparison of Clinical Characteristics and Preoperative Parameters in the Exercise Training Group and Nonexercise Training Group

\begin{tabular}{|c|c|c|c|}
\hline & $\mathrm{EX}(+)$ & $\mathrm{EX}(-)$ & $P$ \\
\hline \multicolumn{4}{|l|}{ Clinical characteristics } \\
\hline Cases & 31 & 33 & NS \\
\hline Age (years) & $54 \pm 3$ & $58 \pm 7$ & NS \\
\hline Gender (male/female) & $21 / 10$ & $19 / 14$ & NS \\
\hline Preoperative class of NYHA & $2.2 \pm 0.7$ & $2.3 \pm 0.8$ & NS \\
\hline Preoperative LVEF (\%) & $59 \pm 3$ & $61 \pm 4$ & NS \\
\hline Atrial fibrillation $(n)$ & 8 & 9 & NS \\
\hline \multicolumn{4}{|l|}{ Underlying disease } \\
\hline Aortic regurgitation $(n)$ & 14 & 14 & NS \\
\hline Mitral regurgitation $(n)$ & 17 & 19 & NS \\
\hline \multicolumn{3}{|l|}{ Procedure } & NS \\
\hline Aortic valve plasty/replacement $(n)$ & $8 / 6$ & $8 / 6$ & NS \\
\hline Mitral valve plasty/replacement $(n)$ & $14 / 3$ & $17 / 2$ & NS \\
\hline \multicolumn{4}{|l|}{ QOL } \\
\hline Nonspecific mental/physical symptom & $11.2 \pm 0.5$ & $10.9 \pm 0.6$ & NS \\
\hline Subjective and social index & $22.7 \pm 1.1$ & $23.1 \pm 1.3$ & NS \\
\hline Total score & $51.6 \pm 1.8$ & $51.7 \pm 2.5$ & NS \\
\hline \multicolumn{4}{|l|}{ Exercise tolerance } \\
\hline Anaerobic threshold $(\mathrm{mL} / \mathrm{min} / \mathrm{kg})$ & $11.4 \pm 0.3$ & $11.5 \pm 0.3$ & NS \\
\hline Peak $\mathrm{VO}_{2}(\mathrm{~mL} / \mathrm{min} / \mathrm{kg})$ & $17.9 \pm 0.8$ & $17.8 \pm 0.8$ & NS \\
\hline \multicolumn{4}{|l|}{ Respiratory function } \\
\hline$\% \mathrm{VC}(\%)$ & $101.7 \pm 2.1$ & $101.0 \pm 2.9$ & NS \\
\hline FEV $1.0 \%(\%)$ & $74.7 \pm 1.4$ & $75.0 \pm 1.5$ & NS \\
\hline
\end{tabular}

EX $(+)=$ exercise training group; EX $(-)=$ nonexercise group; NYHA $=$ New York functional class; $\mathrm{LVEF}=$ left ventricular ejection fraction; FEV = forced expiratory volume; $\mathrm{VO}_{2}=$ oxygen uptake; $\mathrm{NS}=$ not significant.

In addition, CPX, which is a symptom-limited exercise test using an upright bicycle ergometer with expiratory gas analysis, was performed. After a 3 minute rest, warming-up was performed for 3 minutes at 10W, followed by ramp loading (15 W/min). For expiratory gas analysis, an Aeromonitor AE-280S (Minato Inc., Japan) was used. Oxygen uptake at the anaerobic threshold (AT) and maximum oxygen uptake (peak $\mathrm{VO}_{2}$ ) were measured. For the respiratory function test, a Fudac-70 automatic comprehensive respiration-analyzing system (Fukuda Electron Inc., Japan) was used. Percent pulmonary capacity (\%VC) and percent of one second forced expiratory volume (FEV1.0\%) were measured.

For statistical analysis, the unpaired $t$ test, paired $t$ test, chi square test, and variance analysis involving repeated measurement were used. A $P<0.05$ was regarded as significant. The data are expressed as the mean \pm standard deviation. 


\section{RESULTS}

In our questionnaire survey regarding the QOL after open heart surgery, while the improvement rating was generally greater in the exercise training group, there were no significant differences in variance analysis involving repeated measurement. However, the improvement rating, which was calculated from the difference between the preoperative value and the value 6 months after surgery ( $\Delta$ subjective/social index), was significantly greater in the exercise training group than that in the nonexercise group $(4.9 \pm 3.1$ versus $1.1 \pm 4.0, P<0.05)$. Furthermore, the $\Delta$ total score, which was influenced by the above index, was significantly higher in the exercise training group (10.8 \pm 5.3 versus $5.1 \pm 5.2, P<$ 0.05) (Figure 1).
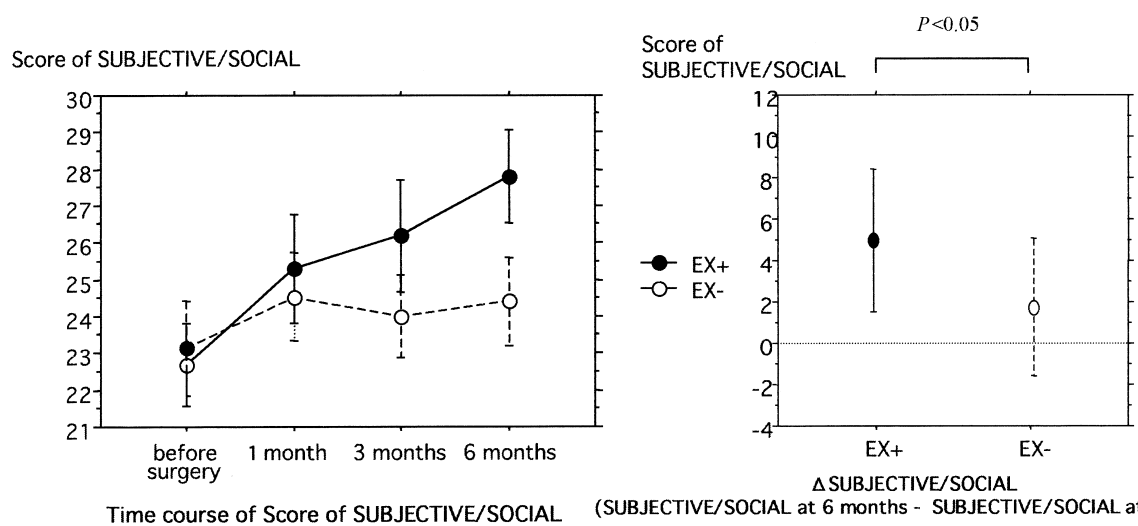

(SUBJECTIVE/SOCIAL at 6 months - SUBJECTIVE/SOCIAL at baseline

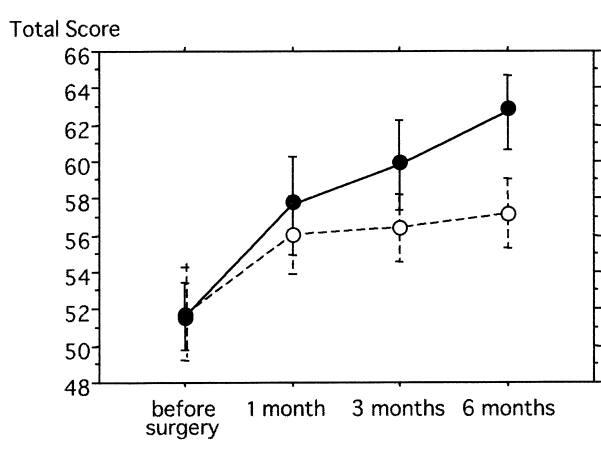

Time course of TOTAL Score

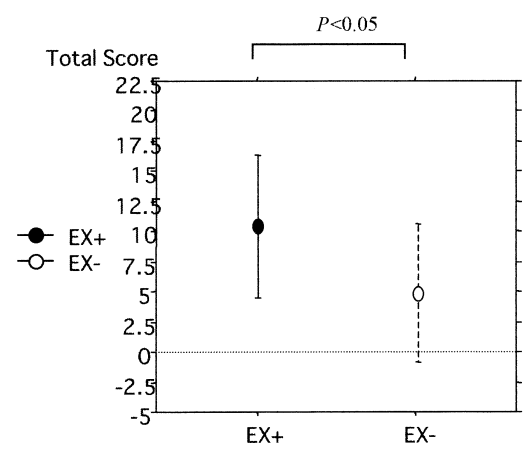

$\triangle$ TOTAL

(TOTAL score at 6 months - TOTAL Score at baseline)

Figure 1. Changes in the questionnaire regarding the QOL.

While the score for the subjective/social index and total QOL score were generally greater in the exercise training group, there were no significant differences in variance (left upper and lower panel). However, the improvement rating ( $\Delta$ subjective/social index), which was calculated from the difference between the preoperative value and the value 6 months after surgery, was significantly greater in the exercise training group than that in the nonexercise group (right upper panel). Furthermore, the $\Delta$ total QOL score, which was influenced by the above index, was significantly higher in the exercise training group (right lower panel). 
The changes in exercise tolerance and respiratory function were also generally greater in the exercise training group. However, there were no significant differences in variance analysis. However, the $\Delta \mathrm{AT}$, which was calculated from the difference between the preoperative value and the value 3 months after surgery, was significantly greater in the exercise training group than that in the nonexercise group $(0.79 \pm 0.17$ versus $-0.02 \pm 0.28, P<0.01)$ (Figure 2$)$. Moreover, there was a positive correlation between the differences in the values of baseline and 6 months after surgery with respect to the subjective/social index and peak $\mathrm{VO}_{2}$ ( $\Delta$ subjective/social index versus $\Delta$ peak $\mathrm{VO}_{2}, \mathrm{r}=0.62, P<0.05$ ) (Figure 3).

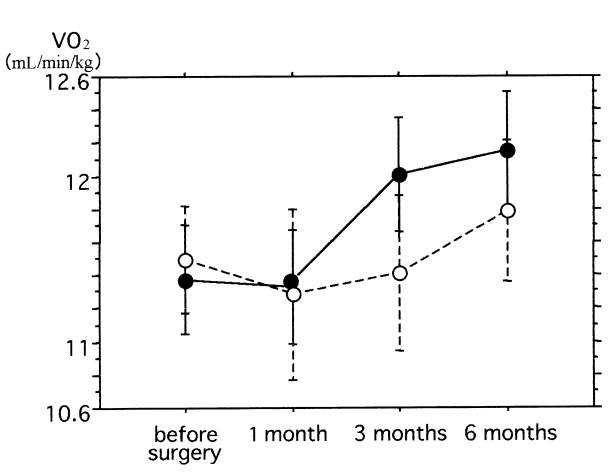

Time Course of Anaerobic Threshold

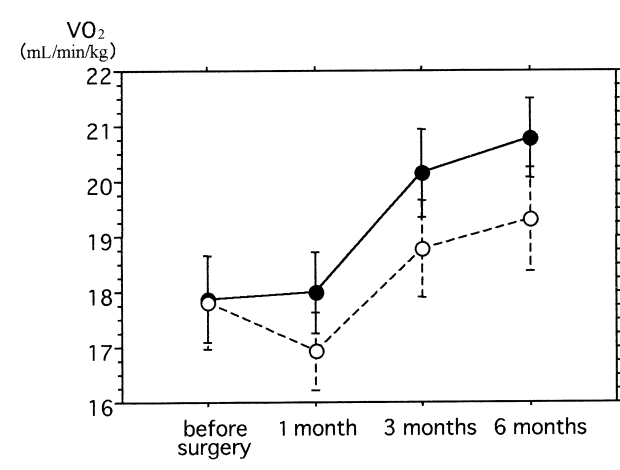

Time Course of Peak VO
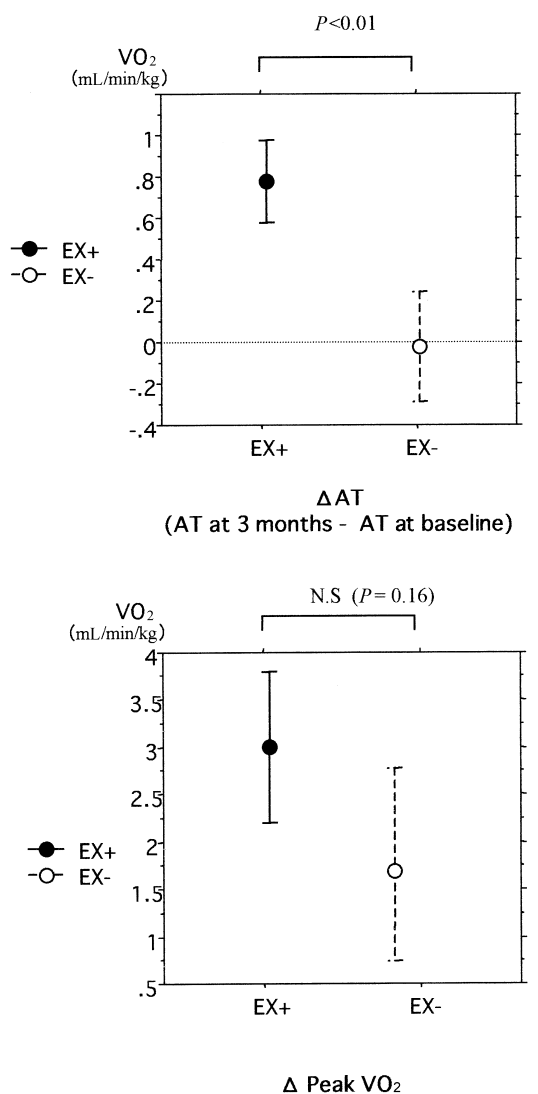

(Peak $\mathrm{VO}_{2}$ at 6 months - Peak $\mathrm{VO}_{2}$ at baseline)

Figure 2. Changes in exercise tolerance.

The changes in exercise tolerance (AT and peak $\mathrm{VO}_{2}$ ) were generally greater in the exercise training group, however, there were no significant differences in variance analysis (left upper and lower panel). While the $\Delta \mathrm{AT}$, which was calculated from the difference between the preoperative value and the value 3 months after surgery, was significantly greater in the exercise training group (right upper panel), there was no significant difference in $\Delta$ peak $\mathrm{VO}_{2}$. 


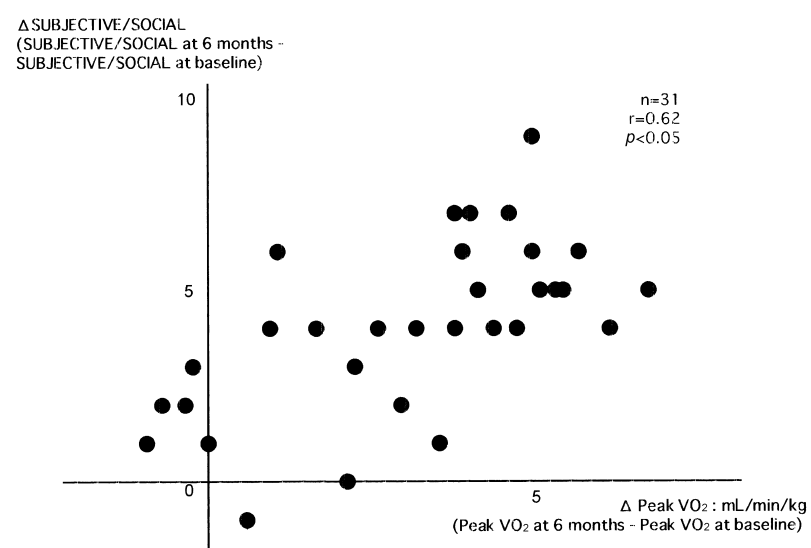

Figure 3. Relationship between $\Delta$ subjective/social index and $\Delta$ peak $\mathrm{VO}_{2}$ in the exercise training group.

There was a positive correlation between the differences in the values at baseline and 6 months after surgery with respect to the subjective/social index and peak $\mathrm{VO}_{2}$ in the exercise training group.

There were no significant differences in the changes for any other obtained parameters, which were calculated from differences between the preoperative values and values 3 or 6 months after surgery, between the two groups.

\section{DISCUSSION}

The treatment for patients with chronic heart failure has previously involved resting as a rule. However, exercise training has been recommended for those patients on the basis of a hypothesis that exercise-related increases in muscle volume, inhibition of the sympathetic nervous system, and enhancement of the parasympathetic nervous system improve cardiac performance. ${ }^{6}$ In the guidelines for the evaluation and management of heart failure, which were established by AHA and ACC special committees, it is stated that "moderate exercise to tolerance should be strongly encouraged"," "Patients should be encouraged to stay as active as possible", and "exercise training as an adjunctive approach to improve clinical status in ambulatory patients". ${ }^{8)}$ While exercise training is aggressively recommended to heart failure patients, several aspects remain to be clarified: subjects for whom exercise therapy should be recommended among patients with chronic heart failure; exercise prescription; interval; and long-term merits. This may apply to patients with valvular disease for which surgery is indicated. However, none of the previous studies have reported the role of postoperative continuous exercise training for these patients in improvement in exercise tolerance and 
the QOL in Japanese subjects. Furthermore, there may be differences in the process of postoperative restoration between a stenotic lesion and a regurgitant lesion. Accordingly, we focused on regurgitant valvular disease.

Medical scales, such as a fall in blood pressure and improvement in left ventricular ejection fraction, can be readily expressed as values. However, QOL in social life cannot always be evaluated. Various methods for evaluating the QOL have been developed. In Japan, in 1990, a questionnaire to evaluate the QOL in Japanese patients with cardiovascular diseases was published by a study group (Director: Jun Fujii) organized by the Ministry of Health and Welfare. ${ }^{5)}$ This questionnaire facilitates the analysis of factors such as disease-related discomfort, life satisfaction, mental stability, life briskness/activity, and life independence. As the content of the questionnaire regarding QOL is extremely subjective and the results are influenced by the value of life and growth process, questionnaires prepared in foreign countries cannot be applied without modifying them. In this respect, the above questionnaire for Japanese is very significant and able to evaluate QOL in Japan.

From the viewpoint of the improvement rating in the QOL questionnaire after open heart surgery, the improvement rating ( $\Delta$ subjective/social index) and $\Delta \mathrm{AT}$, which were calculated from the difference between the values after surgery and the preoperative values, were significantly greater in the exercise training group. Moreover, there was a positive correlation between the $\Delta$ subjective/social index and $\Delta$ peak $\mathrm{VO}_{2}$. It is suggested that changes in the subjective and social index contribute to an exercise training-related improvement in the QOL. In other words, exercise training may favorably affect the mental and spiritual well-being of the patient.

In this study, we administered strictly supervised exercise training to the patients until discharge after surgery, and long-term follow-up was performed. Although there was no significant improvement in respiratory function at rest after discharge until 6 months, the changes in exercise tolerance were similar to those previously reported by us. ${ }^{9)}$

In Japan, specialized institutions and staff responsible for exercise training after discharge are insufficient. Accordingly, it is extremely difficult to properly monitor exercise training. However, cardiac rehabilitation should be applied based on the concept of a long-term comprehensive program consisting not only of exercise training but also education and counseling. Efficient comprehensive rehabilitation for patients for whom it is indicated may be effective.

Limitations: Although ideally clinical studies should be designed as randomized case-controlled studies, this study was designed as a nonrandomized case-controlled study. The exercise training after open heart surgery was thought to be effective and already adapted to our health insurance as an established therapy. A 
randomized case-controlled study, which requires a nonexercise group, was thought to be unethical. The main reason why the patients in the nonexercise group could not be trained was not caused by a medical problem but a social problem in this study. In addition, there were no significant differences in clinical characteristics.

While the $\triangle \mathrm{AT}$ calculated from the difference between the preoperative value and the value for 3 months after surgery was significantly greater in the exercise group than that in the nonexercise group, there was no significant difference in the $\Delta \mathrm{AT}$ calculated from the difference between the baseline value and the value for 6 months after surgery in both groups. During six months, an improvement in postoperative hemodynamics might contribute to an improvement in exercise tolerance even in the nonexercise group. Long-term follow-up may be required to evaluate a long-term effect.

In addition, while "a questionnaire regarding disease and quality of life" was only employed to evaluate QOL in this study, recently, Short Form 36, ${ }^{10}$ ) which was published in Japanese, and a questionnaire regarding physical activities $(\mathrm{SAS})^{11)}$ have been used in clinical practice. In the future, these questionnaires should be more widely used.

Conclusions: Exercise training in patients with valvular disease after open heart surgery may improve their QOL and exercise tolerance. It is suggested that changes in subjective and social parameters contribute to an exercise trainingrelated improvement in the QOL.

\section{REFERENCES}

1. Shephard RJ, Franklin B. Changes in the quality of life: a major goal of cardiac rehabilitation. J Cardiopulm Rehabil 2001; 21: 189-200. (Review)

2. Cohen RA, Moser DJ, Clark MM, et al. Neurocognitive functioning and improvement in quality of life following participation in cardiac rehabilitation. Am J Cardiol 1999; 83: 1374-8.

3. Giannuzzi P, Temporelli PL, Corra U, Tavazzi L: ELVD-CHF Study Group. Antiremodeling effect of longterm exercise training in patients with stable chronic heart failure: results of the Exercise in Left Ventricular Dysfunction and Chronic Heart Failure (ELVD-CHF) Trial. Circulation 2003: 108; 554-9.

4. Weber KT, Janicki JS. Cardiopulmonary exercise testing for evaluation of chronic cardiac failure. Am J Cardiol 1985; 55: 22A-31.

5. Kayaba K, Fujii J. The development of quality of life scales for Japanese patients with cardiovascular diseases. JACD 1990; 25: 89-96.

6. Pina IL, Apstein CS, Balady GJ, et al. Exercise and heart failure: A statement from the American Heart Association Committee on exercise, rehabilitation, and prevention. Circulation 2003; 107: 1210-25. (Review)

7. Grady KL, Dracup K, Kennedy G, et al. Team management of patients with heart failure: A statement for healthcare professionals from The Cardiovascular Nursing Council of the American Heart Association. Circulation 2000; 102: 2443-56.

8. Hunt SA, Baker DW, Chin MH, et al. ACC/AHA Guidelines for the Evaluation and Management of Chronic Heart Failure in the Adult: Executive Summary A Report of the American College of Cardiology/American Heart Association Task Force on Practice Guidelines (Committee to Revise the 1995 Guidelines for the Evalu- 
ation and Management of Heart Failure): Developed in Collaboration With the International Society for Heart and Lung Transplantation; Endorsed by the Heart Failure Society of America. Circulation 2001; 104: 29963007.

9. Sato S, Kamata J, Ueshima K, et al. Changes in respiratory response to exercise before and after cardiac surgery. Cardioangiology 1998; 44: 469-72. (Japanese)

10. Fukuhara S, Bito S, Green J, Hsiao A, Kurokawa K. Translation, adaptation, and validation of the SF-36 Health Survey for use in Japan. J Clin Epidemiol 1998; 51: 1037-44.

11. Sasayama S, Asanoi H, Kihara Y, et al. Clinical effects of long-term administration of pimobendan in patients with moderate congestive heart failure. Heart Vessels 1994; 9: 113-20. 